\title{
ATRIBUTOS FÍSICOS DE LATOSSOLO AMARELO DISTRÓFICO, SOB DIFERENTES FONTES E DOSES DE FÓSFORO
}

lanna Bizerra Barros'; Willian Nogueira de Sousa²; Nayane Fonseca Brito ${ }^{3}$; Iolanda Maria Soares Reis"; Carlos Alberto Costa Veloso5; Arystides Resende Silva6; Eduardo Jorge Maklouf Carvalho7.

\author{
1 Universidade Federal de Viçosa, Minas Gerais, Brasil, iannabb@hotmail.com \\ 2 Universidade Federal do Oeste do Pará, Santarém, Pará, Brasil, wnsagro@gmail.com \\ ${ }^{3}$ Universidade Federal do Oeste do Pará, Santarém, Pará, Brasil, nayanebrito4@gmail.com \\ ${ }^{4}$ Universidade Federal do Oeste do Pará, Santarém, Pará, Brasil, iolandareis@outlook.com \\ ${ }^{5}$ Empresa Brasileira de Pesquisa Agropecuária, Belém, Pará, Brasil, carlos.veloso@embrapa.br \\ ${ }^{6}$ Empresa Brasileira de Pesquisa Agropecuária, Belém, Pará, Brasil, arystides.silva@embrapa.br \\ ${ }^{7}$ Empresa Brasileira de Pesquisa Agropecuária, Belém, Pará, Brasil, eduardo.maklouf@embrapa.br
}

RESUMO: A qualidade física dos solos é fator determinante ao desempenho das culturas de grãos, tendo em vista que alterações em seus atributos resulta em um ambiente adverso ao desenvolvimento do sistema radicular de plantas. O objetivo desse trabalho foi avaliar a influência de fontes e doses de fósforo nos atributos físicos do solo. $O$ estudo foi realizado em Latossolo Amarelo distrófico argiloso em delineamento de blocos casualizados, no esquema fatorial $3 \times 5$ em três blocos, sendo três fontes (Superfosfato simples, Superfosfato triplo e Arad) e cinco doses $(0,60,120$, 180 e $240 \mathrm{~kg} \mathrm{ha}^{-1}$ )de fósforo. Foram determinadas as seguintes propriedades físicas do solo: densidade do solo (Ds), porosidade total (PT), umidade gravimétrica (UG), densidade de partículas (Dp), em três profundidades. As doses de fósforo influenciaram os atributos físicos do solo Ds e PT.

PALAVRAS - CHAVE: Adubação fosfatada, Manejo do solo, Propriedades físicas.

\section{PHYSICAL ATTRIBUTES OF DISTROPHIC YELLOW LATOSOL, UNDER DIFFERENT SOURCES AND PHOSPHORUS DOSES}

ABSTRACT: The soils physical quality is a determinant factor for the performance of grain crops. The objective of this work was to evaluate the influence of sources and doses of phosphorus on the physical attributes of a soil. The study was carried out in a clayey dystrophic Yellow Latosol in a randomized block design, in a $3 \times 5$ factorial scheme in three blocks, three sources (single superphosphate, triple superphosphate and Arad) and five doses $\left(0,60,120,180\right.$ and $\left.240 \mathrm{~kg} \mathrm{ha}^{-1}\right)$ of phosphorus. The following physical soil properties were determined: soil density (Ds), Total Porosity (PT), Gravimetric Humidity (UG), Particle Density (Dp) in three depths. The doses of phosphorus influenced the physical attributes of the soil Ds e PT.

KEYWORDS: Phosphate fertilization, Physical properties, Soil management. 


\section{ATRIBUTOS FÍSICOS DE LATOSSOLO AMARILLO DISTRÓFICO, BAJO DIFERENTES FUENTES Y DOSIS DE FÓSFORO}

RESUMEN: La calidad física de los suelos es un factor determinante en el desempeño de los cultivos de granos, teniendo en cuenta que los cambios en sus atributos resultan en un ambiente adverso al desarrollo del sistema radicular de plantas. El objetivo de este trabajo fue evaluar la influencia de fuentes y dosis de fósforo en los atributos físicos del suelo. El estudio fue realizado en Latossolo Amarillo distrófico arcilloso en delineamiento de bloques casualizados, en el esquema factorial $3 \times 5$ en tres bloques, siendo tres fuentes (Superfosfato simple, Superfosfato triple y Arad) y cinco dosis $(0$, $60,120,180$ y 240) $\mathrm{kg} \mathrm{ha}^{-1}$ ) de fósforo. Se determinaron las siguientes propiedades físicas del suelo: densidad del suelo (Ds), porosidad total (PT), humedad gravimétrica $(U G)$, densidad de partículas (Dp), en tres profundidades. Las dosis de fósforo influenciaron los atributos físicos del suelo Ds y PT.

PALABRAS CLAVE: Fertilización fosfatada, Manejo del suelo, Propiedades físicas.

Os solos brasileiros, especialmente os de regiões tropicais, são característicos por apresentarem baixa fertilidade natural, devido ao alto grau de intemperismo sofrido por eles, sendo o fósforo um dos principais limitantes a produção agrícola em virtude de sua baixa disponibilidade, desse modo práticas de adubação corretiva são necessárias para suprir a necessidade das culturas (NOVAIS et al., 2007).

Fertilizantes fosfatados são os mais comercializados para uso agrícola no Brasil, podendo ser encontrado no comércio sob diferentes concentrações e solubilidades. As fontes solúveis, como é o caso do Superfosfato Triplo (ST), tem apresentado maior eficiência na disponibilidade de fósforo com maior produção de massa seca e incremento desse nutriente no tecido de plantas, quando comparado aos fosfatos naturais (RAMOS et al., 2009; SILVA et al., 2009; FRANDOLOSO et al., 2010; FONTOURA et al., 2010; SILVA et al., 2011). No entanto, tem-se observado que os fosfatos reativos naturais quando aplicados parcelados no sulco 
de plantio apresentam eficiência semelhante aos solúveis, além de proporcionarem maior efeito residual e terem menor custo de aquisição (RESENDE et al., 2006).

A qualidade física dos solos é outro fator determinante ao desempenho das culturas de grãos, onde valores elevados de densidade do solo reduzem o volume de macroporos bem como a taxa de infiltração de água (SANTI et al., 2012), que resultam em perdas significativas na produtividade de culturas como feijão (MONTANARI et al., 2010), milho e soja. Densidades entre 1,32 e 1,35 kg $\mathrm{dm}^{-3}$ proporcionam reduções $27 \%$ no diâmetro médio de raízes de milho e aumento de 49\% em raízes de soja, constatando que a resposta a compactação varia de acordo com espécie cultivada (VALADÃO et al., 2015).

Estudos associando fontes e doses de fósforo nas propriedades físicas do solo ainda são muito incipientes. Os poucos estudos existentes demostram que altas doses de fósforo em solos compactados atuam como condicionantes ao desenvolvimento radicular das plantas, o que permite a exploração de maior volume de solo (RIBEIRO et al., 2010; VALADÃO et al., 2015). Desse modo, o presente trabalho parte da hipótese que fontes e doses de fósforo podem influenciar os atributos físicos do solo.

Diante do exposto, o trabalho teve como objetivo avaliar a influência de fontes e doses de fósforo nos atributos físicos de um Latossolo Amarelo distrófico.

O experimento foi realizado no campo experimental da Embrapa Amazônia Oriental, localizado no município de Belterra - PA, sob as coordenadas geográficas $2^{\circ} 41^{\prime}$ e 25,7" S e $54^{\circ}$ 55' 18,4" W. O clima da região, segundo a classificação de Köppen, é do tipo tropical e entre os meses de julho de 2015 a julho de 2016 apresentou precipitação pluvial média mensal de $87 \mathrm{~mm}$ e temperaturas máximas e mínimas mensais de $29^{\circ} \mathrm{C}$ 
e $20{ }^{\circ} \mathrm{C}$, respectivamente (INMET 2017). O solo da área foi classificado como Latossolo Amarelo distrófico, textura argilosa (Tabela 1), antes da instalação do experimento.

O delineamento experimental foi em blocos casualizados, arranjados em esquema fatorial $3 \times 5$, com três repetições. Foram utilizadas três fontes de fósforo: Superfosfato Simples (SS), Superfosfato Triplo (ST) e fosfato reativo natural (Arad) e cinco doses 0; 60; 120; 180 e 240 kg ha-1 de $\mathrm{P}^{2} \mathrm{O}^{5}$. O Arad foi aplicado a lanço e os superfosfatos triplo e simples tiveram aplicação localizada.

Tabela 1. Caracterização química e granulométrica do solo, antes da instalação do experimento.

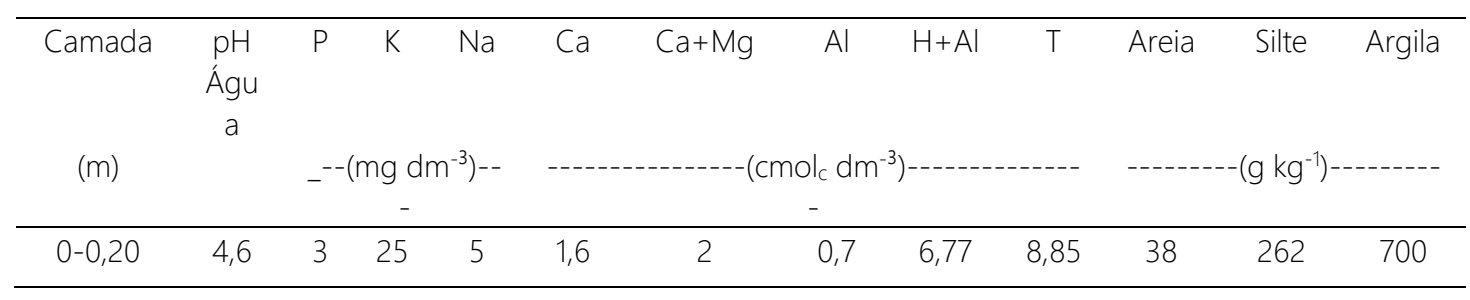

As parcelas experimentais foram de $5 \times 8 \mathrm{~m}$, constituindo área total de $40 \mathrm{~m}^{2}$ e área útil de $14,4 \mathrm{~m}^{2}$. Foi utilizado sementes de milho espaçadas a 0,50 m entre linhas, com três sementes por metro linear, em um total de 240 plantas por parcela. A cultivar utilizada no plantio foi o AG 7088 PROX, sendo semeada, mecanicamente, no dia 03 de março de 2016, para implantação do Sistema de Plantio Direto (SPD). As parcelas, exceto o tratamento controle, receberam adubação mineral com cloreto de potássio e uréia, onde foram aplicados $120 \mathrm{~kg} \mathrm{ha}^{-1}$ de N e $90 \mathrm{~kg} \mathrm{ha}^{-1}$ de $\mathrm{K}_{2} \mathrm{O}$, respectivamente, parcelados em semeadura e cobertura.

Após a colheita do milho, amostras de solos deformadas e indeformadas foram coletadas através de pá e trado tipo TRAI, respectivamente, nas profundidades de 0,00-0,05 m; 0,05- 
0,10 m; 0,10 - 0,20 m, para fins de determinação das propriedades físicas do solo, sendo que para estabilidade de agregados foram utilizadas amostras de profundidade $0,00-0,20 \mathrm{~m}$.

As propriedades avaliadas foram densidade do solo (Ds), pelo método do anel volumétrico, porosidade total (PT) pela relação entre densidade do solo e densidade de partículas (Dp), determinada pelo método do balão volumétrico e umidade gravimétrica (UG), mensurada através da expressão: $100(a-b) / b$, onde "a" corresponde ao peso da amostra úmida e "b" ao peso da amostra seca a $105^{\circ} \mathrm{C}$, sendo as análises realizadas de acordo com os métodos descritos por Embrapa (2009).

Os dados foram submetidos à análise de variância, quando atendidos aos pressupostos teóricos de normalidade e homogeneidade de variância e as médias comparadas pelo teste SNK a 5\% de probabilidade. Foi utilizado o teste não paramétrico de Friedman quando algumas das exigências do modelo paramétrico foram violadas. As análises foram efetuadas com o auxílio de software ASSISTAT 7.7. Foram empregadas a análise de regressão polinomial, através do software Excel.

As fontes e doses de fósforo não apresentaram interação significativa nos atributos físicos do solo, em diferentes profundidades.

As doses de fósforo apresentaram influência sobre a Ds, cujo maior valor encontrado foi de $1,03 \mathrm{~g} \mathrm{~cm}^{-3}$, sob a dose zero (tratamento controle), na profundidade 0, 10 - 0,20 m (Figura 1). Esse resultado está relacionado a algum fator ocasional antes da instalação do experimento, tendo em vista o curto período de condução e o fato da adubação ter sido realizada em superfície, onde as possíveis alterações ocorreriam somente na camada superficial (COSTA et al., 2011). Valadão et al. (2015) encontraram resultados divergentes do presento trabalho, ao observarem que a adubação fosfatada não apresentou influência sobre os atributos físicos do solo. Já as fontes de fósforo não alteraram a Ds. 
Em condições de solo compactado, o fósforo pode atuar como condicionante, ou seja, maiores teores proporcionam incremento em produtividade, atenuando os efeitos negativos da compactação (RIBEIRO et al., 2010; VALADÃO et al., 2015).

A PT diferiu quanto às doses de fósforo aplicadas, onde a maior quantidade de poros $(66,96 \%)$ ocorreu sob a dose de $180 \mathrm{~kg} \mathrm{ha}^{-1}$ de $\mathrm{P}_{2} \mathrm{O}_{5}$, na profundidade de 0,00 - 0,05 m (Figura 2). Assim como na Ds, tal fato pode estar relacionado a algum fator aleatório antes da instalação do experimento (COSTA et al., 2011). Valadão et al. (2015), não observaram influência das doses de fósforo sobre a PT. Já as fontes de fósforo não influenciaram a PT.

A DP e UG não diferiram em função das fontes e doses de fósforo aplicadas. Tais resultados corroboram os encontrados por Valadão et al. (2015), ao não encontrarem influência do fósforo sobre atributos físicos do solo.

Figura 1. Densidade do solo em função das doses de fósforo, nas profundidades 0,00 $-0,05(5) ; 0,05-0,10(10) ; 0,10-0,20 \mathrm{~m}(20)$.

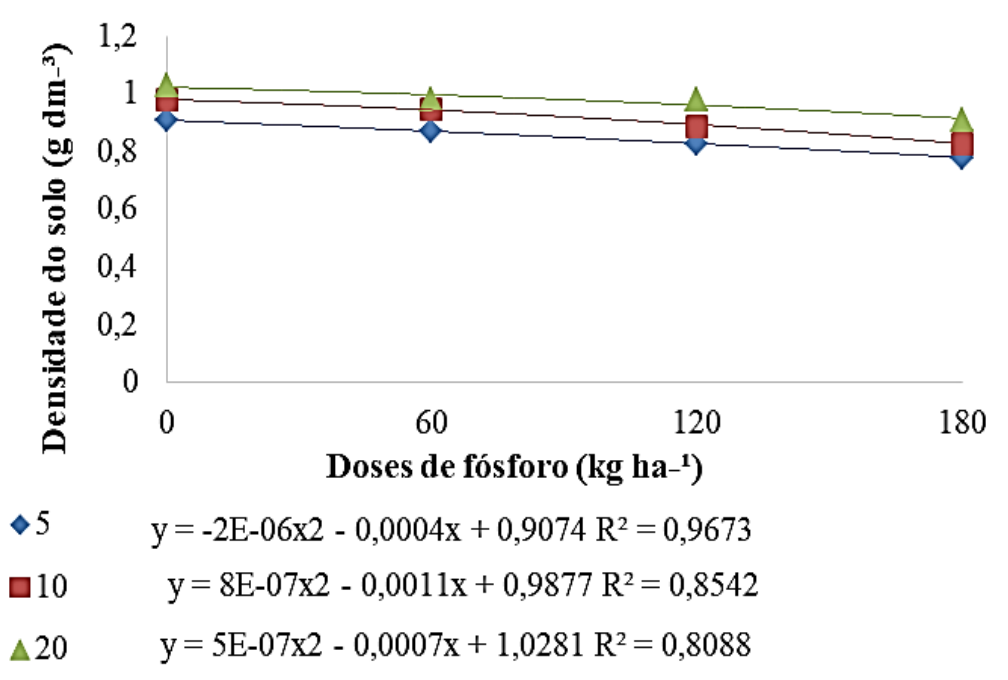

Fonte: Elaborado pelo autor. 
Figura 2. Porosidade total em função das doses de fósforo, nas profundidades 0,00 $0,05(5) ; 0,05-0,10(10) ; 0,10-0,20 \mathrm{~m}(20)$.

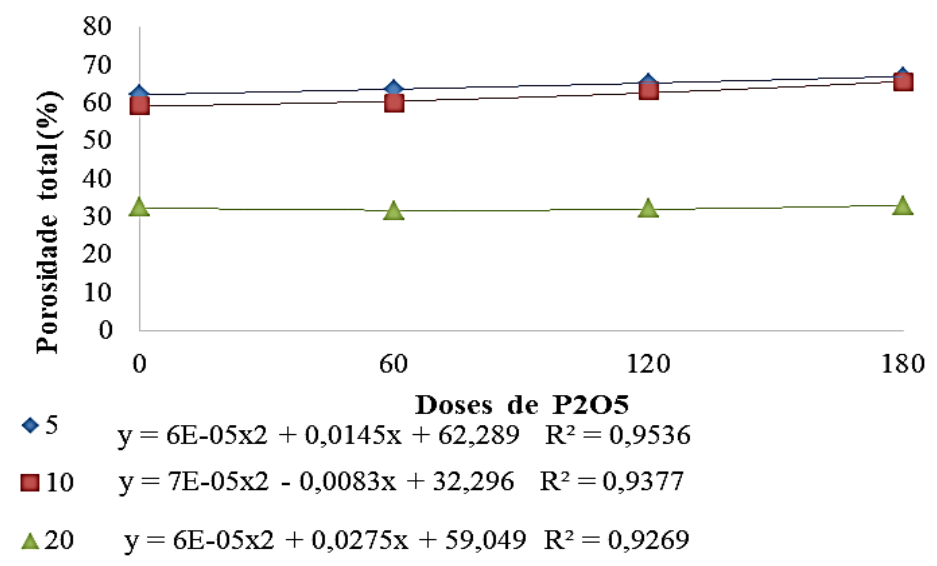

Fonte: Elaborado pelo autor.

No presente estudo a avaliação no primeiro ano de cultivo, para posterior consolidação de sistema de plantio direto, não foi suficiente para mensurar os impactos do manejo e adubação fosfatada nos atributos físicos do solo.

As doses de fósforo influenciaram positivamente os atributos físicos do solo Ds e PT.

\section{REFERÊNCIAS}

COSTA, M. S. S. de M.; PIVETTA, L. A.; COSTA, L. A. M.; PIVETTA, L. G.; CASTOLDI, G.; STEINER, F. Atributos físicos do solo e produtividade do milho sob sistemas de manejo e adubações. Revista Brasileira de Engenharia Agrícola e Ambiental, v. 5, n. 8, p. 810 -
815, 2011.

EMPRESA BRASILEIRA DE PESQUISA AGROPECUÁRIA - EMBRAPA. Centro Nacional de Pesquisa de Solos. Manual de análises químicas de solo, plantas e fertilizantes. $2^{\mathrm{a}}$ ed., Brasília, 2009. 627p.

FONTOURA, S. M. V.; VIEIRA, R. C. B.; BAYER, C.; ERNANI, P. R.; MORAES, R. $P$.; Eficiência técnica de fertilizantes fosfatados em Latossolo sob plantio direto. Revista Brasileira de Ciência do Solo, v. 34, p. 1907 - 1914, 2010.

FRANDOLOSO, J. F.; LANA, M. C.; FONTANIVA, S.; CZYCZA, R, V. Eficiência de adubos fosfatados associados ao enxofre elementar na cultura do milho. Revista Ceres, v. 57, n. 5, p. $686-694,2010$.

INSTITUTO NACIONAL DE METEOROLOGIA (INMET). Disponível 
em:

$<$ http://

http://www.inmet.gov.br/projetos/rede /pesquisa/gera_serie_txt_mensal.php?\& mRelEstacao $=82246 \&$ btnProcesso $=$ seri e\&mRelDtInicio=01/07/2015\&mRelDtFi

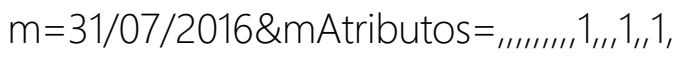
,>. Acesso em 10 de jan. de 2016.

MONTANARI, R.; CARVALHO, M. P.; ANDREOTTI, M.; DALCHIAVON F. C.; LOVERA, L. H.; HONORATO, M. A. O. Aspectos da produtividade do feijão correlacionados com atributos físicos do solo sob elevado nível tecnológico de manejo. Revista Brasileira de Ciência do Solo, v. 34, p. $1811-1822,2010$.

NOVAIS, R. F.; ALVAREZ, V. V. H.; BARROS, N. F.; FONTES, R. L. F.; CANTARUTII, R. B. Fertilidade do solo. Viçosa, MG, Sociedade Brasileira de Ciência do Solo, 2007. p. 471 - 550.

RAMOS, S. J.; FAQUIN, V.; RODRIGUES, C. R.; Silva, C. A.; BOLDRIN, P. F. Biomass production and phosphorus use of forage grasses fertilized with two phosphorus sources. Revista Brasileira de Ciência do Solo, v. 33, p. 335 - 343, 2009.

RESENDE, A. V.; NETO, A. E. F.; ALVES, V. M. C.; MUNIZ, J. A.; CURI, N.; FAQUIN, V.; KIMPARA, D. I.; SANTOS, J. Z. L.; CARNEIRO, L. F. Fontes e modos de aplicação de fósforo para milho em solo cultivado da região do cerrado. Revista Brasileira de Ciência do Solo, v. 30, p. 453 - 466, 2006.

RIBEIRO, M. A. V. NOVAIS, R. F.; FAQUIN, $V_{\text {; }}$ FERREIRA, M.; FURTINI NETO, A. E.; LIMA, J. M.; VILLANI, E. M.
A. Resposta da soja e do eucalipto ao aumento da densidade do solo e a doses de fósforo. Revista Brasileira de Ciência do Solo, v. 34, p. 1157 - 1164, 2010.

SANTI, A. L.; AMADO, T. J. C.; CHERUBIN, M. R.; MARTIN, T. N.; PIRES, J. L.; FLORA, L. P. D.; BASSO, C. J. Análise de componentes principais de atributos químicos e físicos do solo limitantes à produtividade de grãos. Pesquisa Agropecuária Brasileira, v. 37, n. 9, p. 1346 - 1357, 2012.

SILVA, F. N.; FURTINI NETO, A. E.; CARNEIRO, L. F.; MAGALHÃES, C. A. S.; CARNEIRO, D. N. M. Crescimento e produção de grãos de soja sob diferentes doses e fontes de fósforo em solos distintos. Ciência e Agrotecnologia, v. 33, n. 5, p. $1220-$ 1227, 2009.

SILVA, T. O.; FURTINI NETO, A. E.; CARNEIRO, L. F.; PALUDO, V. Plantas de cobertura submetidas a diferentes fontes de fósforo em solos distintos. Semina: Ciências Agrárias, v. 32, n. 4, p. 1315 - 1326, 2011.

VALADÃO, F. C. de A.; WEBER, O. L. S.; VALADÃO JÚNIOR, D. D.; SCAPINELLI, A.; DEINA, F. R.; BIANCHINI, A. Adubação fosfatada e compactação do solo: sistema radicular da soja e do milho e atributos físicos do solo. Revista Brasileira de Ciência do Solo, v. 39, p. $243-255,2015$. 